\title{
Movimientos sociales, acción colectiva y cambio social en perspectiva. Continuidades y cambios en el estudio de los movimientos sociales. Rubén Díez García y Gomer Betancor Nuez (eds.). Mendiola: Fundación Betiko, 2019, 226 pp. ISBN: 978-84-09-09287-1
}

\author{
Andy Eric Castillo Patton \\ Departamento de Antropología Social y Psicología Social, Universidad Complutense de Madrid. España / Spain \\ andcas03@ucm.es
}

Más de ocho años después de la eclosión del $15 \mathrm{M}$ y de los "novísimos" movimientos sociales, los social movement studies parecieran estar viviendo una Edad de Plata 0, incluso, una Segunda Edad de Oro. Dicha afirmación, aunque pudiera resultar osada, se fundamenta, en primer lugar, en un elevado número de publicaciones e investigaciones que inundan multitud de debates, desbordando los límites de lo exclusivamente académico. En segundo lugar, se encontraría la sobresaliente calidad de gran cantidad de estas publicaciones, no tanto por su posición en los rankings de revistas científicas de impacto, sino por el sugerente contenido que las caracteriza. Este segundo hecho se atestigua en la obra colectiva Movimientos sociales, acción colectiva y cambio social en perspectiva. Continuidades y cambios en el estudio de los movimientos sociales, coordinada por Rubén Díez García (UCM) y Gomer Betancor Nuez (UNED). Esta obra supone una compilación de ensayos e investigaciones vibrantes y excelentes en las materias que ocupa el título de la publicación: los movimientos sociales, la acción colectiva y el cambio social. Teniendo presente el espacio que da origen a esta obra, el Encuentro Intercongresual del Comité de Investigación (Cl-20) de Movimientos Sociales, Acción Colectiva y Cambio Social de la Federación Española de Sociología celebrado el 31 de mayo y el 1 de junio de 2018 en Madrid, en el Centro de Investigaciones Sociológicas y Facultad de Ciencias Políticas y Sociología de la UNED, encontramos que el espíritu de diálogo e intercambio de ideas de este encuentro científico cristaliza en el presente volumen, dividido en cinco bloques y compuesto por trece capítulos, más la introducción. En cuanto a la estructura del texto, los bloques comprenden cinco ejes fundamentales que marcaron el contenido científico del Encuentro Intercongresual, y que pivotan en torno a los principales cambios acaecidos en el estudio de la protesta y de la política no convencional 0 extrainstitucional.

El primer bloque ("Cambios recientes en la protesta y las movilizaciones") lo inaugura Tomás Alberich con un análisis retrospectivo e introspectivo del $15 \mathrm{M}$ y su impacto más inmediato en la conformación y/o refuerzo de otros actores de base (pp. 17-24); a continuación, Antonio Antón presenta una propuesta crítica, pero constructiva, centrada en el marco interpretativo de la teoría populista, fundamental para entender las nuevas fórmulas de "partido-movimiento" en España (pp. 31-42); seguidamente, Ion Andoni del Amo invita a repensar los marcos analíticos de la propia sociología política, poniendo en contexto el creciente fenómeno de la desglobalización, las tensiones que suscita y las reconfiguraciones de la subjetividad en la era de la pospolítica neoliberal (pp. 43-47); cerrando este primer bloque, Antonio Álvarez-Benavides introduce el análisis sobre los nuevos actores de la extrema derecha española en su vertiente más movimentista y de base, con repertorios de protesta similares a los de los movimientos sociales más progresistas (pp. 59-69).

El segundo bloque ("Transformaciones y novedades en las movilizaciones feministas"), dedicado a los feminismos en España, lo abre Adriana Razquin con una mirada etnográfica situada en el origen de la ruptura-transformación del feminismo de 
nueva ola con el $15 \mathrm{M}$ y la posterior deriva del movimiento de los indignados hacia posicionamientos adscritos a la subjetividad patriarcal (pp. 73-86); a continuación, y en conexión con este último capítulo, Carmen Galdón ofrece una reconstrucción de los hitos y los puntos de inflexión que los feminismos en España han contemplado entre el 15M de 2011 y el 8M de 2017, sobre todo la apertura al diálogo intergeneracional y cómo los feminismos desembarcaron en espacios antes limitados a su interlocución (pp. 87-110).

El tercer bloque ("Viejos y nuevos conflictos. Cambios de contexto y nuevas generaciones de activistas"), enfocado en los procesos de creación y transformación de las nuevas generaciones de activistas, es encabezado por el análisis de Francisco Fernández-Trujillo sobre las incipientes nuevas formas de sindicalismo movimentista (social movement unionism) en España que, de acuerdo con el autor, serían fruto de fuertes reconfiguraciones en el mundo del trabajo y la coincidente crisis de representación de los sindicatos tradicionales (pp. 103116); posteriormente, Antonio Montañés y Antonio Álvarez-Benavides reflexionan acerca del proceso de mutación de activistas de nuevo cuño en cargos políticos institucionalizados, abordando el caso del colectivo Juventud Sin Futuro (pp. 117-129).

El cuarto bloque ("Movimientos sociales en el espacio. Una mirada internacional") está dedicado al estudio de los movimientos sociales fuera de España, sobre todo desde una perspectiva que interpela a un análisis desde el territorio de lo físico y de lo simbólico. Este bloque lo estrena Juan Pablo Paredes con un análisis pormenorizado de la genealogía y el legado del movimiento estudiantil en Chile, clave para entender la actual repolitización de la sociedad chilena, despolitizada durante el periodo de la transición a la democracia (neo)liberal (pp. 133-147); por otra parte, Milena Silvester, en relación con el "golpe parlamentario" de 2016 en Brasil, interpela a la reflexión deconstructiva de la lógica que erige al Estado, en particular el Estado neocolonial, en un afán por comprender las tensiones a las que se enfrentan los movimientos sociales emancipatorios en su interacción con estructuras de poder marcadas por las lógicas de la necropolítica (pp. 149-159); por último, Valentín Clavé-Mercier, desde una original aproximación a la geografía política, estudia el movimiento soberanista maorí, poniendo en evidencia la importancia de la dimensión geográfica-espacial en el estudio general de los movimientos sociales, y no solo teniendo presente la tan frecuente dimensión histórico-temporal (pp. 161-173).

El quinto bloque ("Resultados de los movimientos sociales en perspectiva histórica"), a modo de cierre del volumen, propone una reflexión acerca de la trayectoria del estudio de los movimientos sociales por parte de la sociología en las últimas décadas, sobre todo desde el prisma académico español. En el primer capítulo de este último bloque, Susana Aguilar nos acerca al examen del escaso impacto que ha tenido la perspectiva del policy analysis en el estudio de la efectividad real de los movimientos sociales en relación a una materialización política, legislativa 0 judicial de sus demandas (pp. 177192). Por último, Benjamín Tejerina, María Jesús Funes y Ramón Adell, moderados por Gomer Betancor Nuez y Rubén Díez García, protagonizan un debate transcrito acerca de la evolución del estudio de los movimientos sociales (o "acciones colectivas de base") en España desde la etapa de la Transición. En los disensos y consensos establecidos en el conversatorio se observa que el campo de estudio de los movimientos sociales vive un momento de máxima efervescencia en el panorama nacional e internacional, siendo explícitamente evidente el proceso por el cual la academia española ha logrado equipararse, en relación a marcos de interpretación sólidos y producción científica de impacto, con las academias europea e internacional, con las cuales ha logrado establecer importantes lazos e intercambios intelectuales (pp. 193-216).

Una vez vista la estructura elemental de la publicación y de los capítulos que lo componen, podríamos argumentar que las interpretaciones que la vertebran están atravesadas por un denominador común a todas las miradas: la consolidación del giro culturalista en los social movement studies. Esta apreciación se fundamenta en el nexo que se puede establecer entre los diferentes capítulos en base a unos análisis centrados en la dimensión más subjetivista de los movimientos sociales. Entre estos focos analíticos encontraríamos el estudio de los imaginarios que interpelan 0 activan a los sujetos, el vector identitario que los caracteriza, los 
símbolos que manejan, la gestión de la memoria colectiva y el legado histórico, la lógica detrás de los nuevos y viejos repertorios de protesta, el tipo de redes que se constituyen entre activistas, etcétera. Esta aproximación encajaría con las recomendaciones que ya enunció agudamente Alain Touraine (2006) en su diagnóstico sobre el cambio de paradigma social en la agotada y agonizante sociedad industrial, donde el componente centrípeto del trabajo ha sido desplazado por la fuerza centrífuga de la cultura, entendida esta última en su concepción semántica más extensa. Este cambio motiva una mutación de las propias subjetividades de los individuos en la sociedad posindustrial, quienes no desmerecen la importancia que aún tiene el trabajo en sus vidas, pero cuyas identidades gozan de un mayor arraigo en patrones culturales 0 de consumo identitario. Estos factores exigen una adaptación de la mirada científica que escudriña a los movimientos sociales y del modo de hacer sociología y ciencia social en general.

En relación con los textos que aparecen en Movimientos sociales, acción colectiva y cambio social en perspectiva. Continuidades y cambios en el estudio de los movimientos sociales, este giro culturalista está magistralmente consumado dado que, a pesar de tratarse de especifidades 0 realidades muy locales o fragmentadas, interpelan a un cosmos general donde los saberes y las luchas sociales se conjugan en una constelación interactiva y multilateral de lo que María Jesús Funes propone denominar como "acciones colectivas de base" (p. 197). Esta apreciación entra en contacto con las recientes propuestas de Geoffrey Pleyers (2019) acerca de la necesidad de una sociología global de los movimientos sociales, cuyos postulados arraigan en la propia obra de Touraine y su propuesta por una sociología crítica del actor. También enlaza con las aportaciones de François Houtart en torno a las lecturas que inspiran al denostado tercermundismo y la importancia de un pensamiento analítico situado, pero abierto al diálogo entre nodos globales. Esto es lo que precisamente se contempla entre los capítulos del presente volumen: un diálogo abierto y "glocal", vertebrado y vibrante, que nos habla, precisamente, del cambio que actualmente acontece en nuestras sociedades actuales. Se vislumbra, por ejemplo, en el texto de Clavé-Mercier, donde el caso del soberanismo maorí y su articulación histórica como movilización social, que se da en base a una politización del espacio-territorio, sirve para entender las lógicas que activan a otros movimientos soberanistas como el saharaui, el quebequense 0 el catalán, por citar unos pocos ejemplos de actualidad. A este respecto, y en relación con el estudio general de los movimientos sociales, esta reflexión sobre lo espacial "permite evitar la visión demasiado extendida de los movimientos sociales de escala nacional o internacional como monolíticos u homogéneos" (p. 167). Sin embargo, la consolidación de este giro cultural en el estudio de los movimientos sociales se observa en la proliferación de la "vía de la subjetividad", tal y como la formula Pleyers (2019), en multitud de movimientos sociales, sean de corte emancipatorio 0 autoritario. En este sentido, un movimiento social no deja de ser un productor, o (re)constructor, de sociedad, sea desde una perspectiva de lo económico, sea desde una perspectiva de lo cultural (aunque ambos espacios, por definición, se encuentran más bien solapados). En relación con lo anterior, los procesos de reproducción social o de ruptura social interpelan a la condición de policy makers o challengers de los movimientos sociales en calidad de motores del cambio de todo el conjunto social, dado el inevitable factor de cotidianeidad de los mismos (Revilla, 1994). Esta aseveración conecta con la idea de que, a pesar de la emergencia o excepcionalidad de su proceso creativo, el movimiento social y la movilización obedecen cada vez más a una dimensión en la que la subjetividad vence al precepto que domina una, ya casi museística, estrategia o racionalidad de masas. Y más si se tiene presente el paradigma de la "sociedad de la información" (Castells, 2005 [1997]), donde la irrupción de las redes sociales digitales en la vida de los sujetos promueve la activación de un pensamiento social muy fragmentado y selectivo, a pesar de una mayor accesibilidad al conocimiento.

Este proceso de tensionamiento se observa en distintos capítulos de los reseñados anteriormente, como, por ejemplo, en el de Álvarez-Benavides, centrado en el resurgimiento de movimientos sociales de extrema derecha en España, antecesores de un actual giro autoritario-identitario de una parte de la sociedad española que se vuelca en 
opciones partidistas como Vox. Por otra parte, ese tensionamiento también se detecta en la nueva crisis social y política que se vive en América Latina, tal y como ayudan a comprender Paredes, en relación al encarnizamiento neoliberal-disciplinario en Chile, y Silvester, en correspondencia con el auge del neofascismo en Brasil. No son territorios 0 movimientos aislados, sino que son respuestas locales a fenómenos globales. Aquí es primordial tener presente la brillantez explicativa del capítulo de Del Amo en relación a cómo las "nuevas formas de movilización espasmódica y los nuevos imaginarios poscrisis de lo común-popular antagónico deben interpretarse en el contexto de los efectos de desarticulación social operados por la globalización del capitalismo tardío y sus formas culturales posmodernas, contra las que espontáneamente en parte reacciona" (p. 54). Esta afirmación conectaría, además, con la apreciación de cómo los procesos de creciente fragmentación autocomunicativa responden, paradójicamente, al proceso de la desglobalización cultural, tal y como ocurre con la guerra comercial entre Estados Unidos y China, el laberinto del Brexit o el resurgir de otros territorialismos o neonacionalismos reactivos. De ahí que sea imprescindible, según apunta Antón, el hecho de que, en términos de marcos analíticos, desde la sociología política y de los movimientos sociales se deba superar inmediatamente "el enfoque populista, del simple antagonismo ligado al idealismo discursivo posmoderno, así como el determinismo economicista, de la sobrevaloración de las estructuras económicas e institucionales que se imponen a la propia gente como actor sociopolítico y conIlevan un inevitable futuro" (p. 40). Una discusión de fundamental importancia para evitar aquello de que Tejerina y Adell avisan: el riesgo de que academia y activismo se hibriden en exceso. No obstante, es importante mantener una fluidez en los diálogos que se pueden mantener entre activistas y académicos (así como en académicos activistas y activistas académicos) dado que, como bien señalan Alberich, Razquin o Galdón, en frecuentes ocasiones es necesaria una mirada analítica desde dentro de los propios movimientos sociales para entender precisamente las lógicas que se movilizan y los itinerarios que estos recorren. Esto es fundamental a la hora de interpelar a nuevos actores cuyas demandas, por poner los ejemplos del movimiento de los indignados y de los feminismos de cuarta ola, representan una extrema disonancia social dada la radicalidad de sus postulados, que minan los cimientos de las sociedades pluralistas y patriarcales contemporáneas, cuyos vestidos de democracia e igualdad formal representan, en realidad, tupidos velos ante multitud de fenómenos que siguen siendo autoritarios en su condición y están atravesados por rígidos mandatos de género.

Por ello, entre otros tantos temas que se podrían discutir al respecto, es extremadamente recomendable la lectura de Movimientos sociales, acción colectiva y cambio social en perspectiva. Continuidades y cambios en el estudio de los movimientos sociales. Una obra colectiva sugerente y vivaz, ágil y diversa, pero notoriamente completa. De ahí la encarecida recomendación de la lectura de estos textos bien por ojos académicos, que deseen profundizar en las materias que se han discutido, bien por ojos más noveles 0 peregrinos en el estudio de los movimientos sociales, cuyo anhelo por empaparse de los debates más recientes no dejará espacio a la quietud, sino que dará paso a la más apasionante de las reflexiones.

\section{REFERENCIAS BIBLIOGRÁFICAS}

Castells, M. [2005 (1997)]. La sociedad red. La era de la información (Vol. 1). Economía, sociedad y cultura. Madrid: Alianza Editorial.

Pleyers, G. (2019). Movimientos sociales en el siglo XXI: perspectivas y herramientas analíticas. Barcelona: Icaria.

Revilla, M. (1994). El concepto de movimiento social: acción, identidad y sentido. Zona Abierta, 69, 181-213.

Touraine, A. (2006). Un nuevo paradigma para comprender el mundo de hoy. Barcelona: Paidós. 